\title{
SEX-LINKED GENES IN AGE-STRUCTURED POPULATIONS
}

\author{
JAMES L. CORNETTE \\ Department of Mathematics, lowa State University, Ames, lowa, U.S.A. 50011
}

Received: 5. viii.77

\begin{abstract}
Summary
We study the progress towards equilibrium of the frequencies of sex-linked genes in elementary discrete time models of age-structured, overlapping generation populations. It is found that, if a finite upper age limit is assumed, the difference in the frequencies of an allele in males and females will oscillate as in the familiar non-overlapping generation models, al though the oscillations may be irregular. Monotonic convergence of that difference, as found by Nagylaki (1975) in continuous-time overlapping generation models without age-structure, occurs in the models considered here only when there is no upper age limit and when there is "sufficient" overlap of generations.
\end{abstract}

\section{INTRODUCTION}

When the frequencies of a gene at a sex-linked locus differ between the males and females of a population, there is gradual progress towards equilibrium between the frequencies in the sexes, in contrast to the case of an autosomal locus in which equilibrium is reached after only one generation of random mating. A familiar result is that, for discrete time, non-overlapping generation populations with random mating and no selection, the difference in the female and male frequencies of a sex-linked gene in generation $t+1$ is minus one-half times the same difference in generation $t$ (Kempthorne, 1969, pp. 30-34, or Crow and Kimura, 1970, pp. 44-47). Thus, the difference oscillates between positive and negative values, and its magnitude is halved in each generation. Recently, however, Nagylaki (1975) found, in a continuous-time model with overlapping generations but without agestructure, that the same difference approaches zero as $e^{-c t}$ and therefore does not oscillate, but approaches zero monotonically. We explore here, in the context of elementary age-structured populations, the distinctions that lead to oscillating or monotonic convergence of this parameter. In our examples, it will be seen that overlapping generations may interrupt the strictly lockstep oscillations between positive and negative values of this parameter, but that, with the assumption of finite maximum age at death, irregular but almost periodic oscillations will persist. Monotonic convergence seems, at least in our models, to be associated with overlapping generation populations in which there is no upper bound on the length of life of individuals in the population.

\section{An elementary Age-STRUCTURED MODEL}

We consider a discrete time, $t=0,1,2, \ldots$, population genetics model of a gene at a single sex-linked locus with two neutral alleles, $A_{1}$ and $A_{2}$,

Journal Paper No. J-8907 of the Iowa Agriculture and Home Economics Experiment Station, Ames, Iowa. Project 1669. Partial support by National Institutes of Health Grant GM 13827. 
in a population with age groups $x=0,1, \ldots, k$ (possibly $k=\infty$ ) and a single life table $l(x), x=0, \mathrm{l}, \ldots, k$ for all genotypes in both sexes. For any sex-genotype category, $l(x)$ is the proportion of individuals of that category in age group 0 at time $t-x$ that survive to age $x$ at time $t$ (implying that $l(0)=1)$. Assume that the population is constant in size and in age distribution in both sexes so that, in either sex, with

$$
A=l(0)+l(1)+\ldots+l(k),
$$

the proportion of individuals of age $x$ at any time is $l(x) / A$. (Allowance could be made for regular geometric growth of the population with growth rate $\gamma$ by computing the age distribution with an adjusted function $l^{*}(x)=l(x) \gamma^{-x}$ (see, for example, Leslie (1945)), but constant population size is sufficient for the present study.) Generally, assume that every parameter is constant in time except the frequencies $p(t, x)$ and $p^{\prime}(t, x)$ of the $A_{1}$ gene among males (the heterogametic sex) and females (the homogametic sex), respectively, in age group $x$ at time $t$. Assume that the fertile period for both males and females is from age $i$ to age $j$, and assume equally fecund random matings between fertile males and fertile females. Let $\vec{\Delta}(t)$ be the vector

$$
\vec{\Delta}(t)=\left[\begin{array}{c}
p^{\prime}(t, 0)-p(t, 0) \\
p^{\prime}(t, 1)-p(t, 1) \\
\vdots \\
p^{\prime}(t, k)-p(t, k)
\end{array}\right]
$$

Then we are interested in the progress in time of $\vec{\Delta}(t)$ and particularly of the quantity

$$
q(t)=\sum_{x=0}^{k}\left(p^{\prime}(t, x)-p(t, x)\right) l(x) / A,
$$

which is the difference of the frequencies of the $A_{1}$ gene in the female and male populations.

Given $\vec{\Delta}(t)$, we compute $\vec{\Delta}(t+1)$ as follows. Let

$$
B=l(i)+l(i+1)+\ldots+l(j) .
$$

Then

$$
\begin{aligned}
p^{\prime}(t+1,0) & =\sum_{x=i}^{j} \sum_{y=i}^{j} \frac{l(x)}{B} \frac{l(y)}{B} \frac{\left(p^{\prime}(t, x)+p(t, y)\right)}{2} \\
& =\frac{1}{2 B} \sum_{x=i}^{j} l(x)\left(p^{\prime}(t, x)+p(t, x)\right) \\
p(t+1,0) & =\frac{1}{B} \sum_{x=i}^{j} l(x) p^{\prime}(t, x) \\
p^{\prime}(t+1, x) & =p^{\prime}(t, x-1), \quad x \geqq 1 \\
p(t+1, x) & =p(t, x-1), \quad x \geqq 1 .
\end{aligned}
$$


Then

and

$$
\vec{\Delta}(t+1)=\left[\begin{array}{c}
-\frac{1}{2 B} \sum_{x=i}^{j} l(x)\left(p^{\prime}(t, x)-p(t, x)\right) \\
p^{\prime}(t, 0)-p(t, 0) \\
p^{\prime}(t, 1)-p(t, 1) \\
\vdots \\
p^{\prime}(t, k-1)-p(t, k-1)
\end{array}\right]
$$

$$
\begin{aligned}
q(t+1)=\frac{1}{A}\left[l(0)\left(\frac{-1}{2 B}\right) \sum_{x=i}^{j} l\right. & l(x)\left(p^{\prime}(t, x)-p(t, x)\right) \\
& \left.+\sum_{x=1}^{k} l(x)\left(p^{\prime}(t, x-1)-p(t, x-1)\right)\right]
\end{aligned}
$$

Remarks. Note that although (1) and (2) were derived on the assumption of random mating between all fertile males and females, precisely the same equations would arise from the assumption that females of age $x$ mated (randomly) only with males of age $x$. It can be seen that the oscillations in $q(t)$ are induced by the factor $-\frac{1}{2} B$ in the first component of $\vec{\Delta}(t)$ (associated with the difference in gene frequencies between newborn females and males) and that the remaining components of $\vec{\Delta}(t+1)$ (the older age groups) provide a potential reservoir of stability that may counter the induced oscillations.

Because there is a single life table for all sex-genotype categories, it follows that, for all values of $t$ and $x$ with $t \geqq x$,

$$
p(t, x)=p(t-x, 0) \text { and } p^{\prime}(t, x)=p^{\prime}(t-x, 0) .
$$

We introduce the function

$$
\begin{aligned}
y(t) & =p^{\prime}(t, 0)-p(t, 0) \quad t \geqq 0 \\
& =p^{\prime}(0,-t)-p(0,-t) \quad t=-1,-2, \ldots,-k .
\end{aligned}
$$

Then, by $(4), \vec{\Delta}(t)$ and $q(t)$ become

$$
\begin{aligned}
\vec{\Delta}(t) & =[y(t), y(t-1), \ldots, y(t-k)]^{T} \\
q(t) & =\sum_{x=0}^{k} l(x) y(t-x) / A,
\end{aligned}
$$

and inspection of the first component of $\vec{\Delta}(t+1)$ in (3) leads to

$$
y(t+1)=-\frac{1}{2 B} \sum_{x=i}^{j} l(x) y(t-x),
$$

\section{MOdels WITH FINITE UPPER AGE LIMIT}

For $k$ finite, we consider first the two extreme cases for choices of $i$ and $j$ $(i=j$ and $i=0, j=k$ ) for which simple arguments establish that $q(t)$ periodically changes sign, and then show that in the general case, with 
only a slight restriction on $l(x), q(t)$ will be asymptotic to a periodically oscillating function.

Case $\dot{\mathrm{i}}=\mathrm{j}$. This case is similar to a non-overlapping generation model (or more accurately, a collection of $i+1$ disjoint, non-overlapping generation populations), and the result is the same. From (6) and (5)

$$
\begin{aligned}
y(t+1) & =\left(-\frac{1}{2}\right) y(t-i), \quad t=0,1,2, \ldots \\
q(t+i+1) & =\sum_{x=0}^{k} l(x) y(t+i+1-x) / A \\
& =\sum_{x=0}^{k} l(x)\left[-\frac{1}{2} y(t-x)\right] / A \\
& =-\frac{1}{2} q(t), \quad \text { for } t \geqq k .
\end{aligned}
$$

Case $\mathrm{i}=0, \mathrm{j}=\mathrm{k}$. Inspection of (6) and (5) yields (note that $A=B$ )

$$
\begin{aligned}
y(t+1) & =-\frac{1}{2 A} \sum_{x=0}^{k} l(x) y(t-x) \\
& =-\frac{1}{2} q(t)
\end{aligned}
$$

It is clear from (7) that if $y(t)$ is positive (negative) for $k+1$ successive values $y\left(t_{0}-k\right), y\left(t_{0}-k+1\right), \ldots, y\left(t_{0}\right)$, then the next value, $y\left(t_{0}+1\right)$, is negative (positive), so that $y(t)$ has both positive and negative values in every time interval of length $k+2$. From (8), $q(t)$ similarly has both positive and negative values in every interval of length $k+2$.

General case. With no restrictions on $i$ and $j$, we think of (6) as a difference equation with characteristic polynomial

$$
\lambda^{j+1}+\frac{1}{2 B} \sum_{x=i}^{j} l(x) \lambda^{j-x}=0
$$

which has roots $\lambda_{0}, \lambda_{1}, \ldots, \lambda_{\mathrm{j}}$, listed in decreasing order of magnitude. For simplicity of discussion, we assume that (9) has no multiple roots. Then the solution to (6) will be

$$
y(t)=c_{0} \lambda_{0}^{t}+c_{1} \lambda_{1}^{t}+\ldots+c_{j} \lambda_{j}^{t}, \quad t \geqq-j,
$$

where the constants $c_{0}, c_{1}, \ldots, c_{j}$ are determined by $y(-j), \ldots, y(0)$, which reflect the differences of the frequencies of the $A_{1}$ allele among females and males in the founder population. Then, for $t \geqq k-j$,

$$
\begin{aligned}
q(t) & =\frac{1}{A} \sum_{x=0}^{k} l(x) \sum_{z=0}^{j} c_{z} \lambda_{z}^{t-x} \\
& =\frac{1}{A} \sum_{z=0}^{j} \lambda_{z}^{t} c_{z} \sum_{x=0}^{k} l(x) \lambda_{z}^{-x} \\
& =\sum_{z=0}^{j} \tilde{c}_{z} \lambda_{z}^{t}
\end{aligned}
$$

Because the coefficients of (9) are positive, (9) has no positive or zero roots. The magnitudes of the roots of a polynomial $z^{n}+a_{1} z^{n-1}+\ldots+a_{n-1} z+a_{0}$ 
are either less than $I$ or less than or equal to $\sum_{i=1}^{n}\left|a_{i}\right|$. In (9), the sum of the corresponding coefficients is $\frac{1}{2}$, so all of the roots of (9) are less than one in magnitude (implying that $q(t) \rightarrow 0$ as $t \rightarrow \infty$ ). We assume a minor restriction on $l(x), x=0,1, \ldots, k$ and the founding population that $\tilde{c}_{0} \neq 0$, and either

(A) $\lambda_{0}$ is negative and $\left|\lambda_{0}\right|>\left|\lambda_{1}\right|$

(B) $\lambda_{0}$ is complex, $\lambda_{1}$ is the conjugate of $\lambda_{0}$ and $\left|\lambda_{0}\right|=\left|\lambda_{1}\right|>\left|\lambda_{2}\right|$.

If $(\mathrm{A})$, then

$$
q(t)=\tilde{c}_{0} \lambda_{0}^{t}\left[1+0\left(\left(\frac{\lambda_{1}}{\lambda_{0}}\right)^{t}\right)\right],
$$

and $q(t)$ is asymptotic to $\tilde{c}_{0} \lambda_{0}^{t}$ which changes algebraic sign in every time interval of length one.

If (B), assume that $\lambda_{0}=\gamma e^{i \theta}, \theta>0$. If $\theta<\pi /(j+1)$, with $\lambda=\lambda_{0}$ in (9) the terms of the LHS have positive complex parts and cannot sum to zero; hence $\theta \geqq \pi /(j+1)$. There are real numbers $a$ and $b$ such that

$$
\begin{aligned}
q(t) & =\tilde{c}_{0} \lambda_{0}^{t}+\tilde{c}_{1} \lambda_{1}^{t}+\sum_{z=2}^{j} \tilde{c}_{z} \lambda_{z}^{t} \\
& =a r^{t} \cos (t \theta+b)+\sum_{z=2}^{j} \tilde{c}_{z} \lambda_{z}^{t} \\
& =a r^{t}\left[\cos (t \theta+b)+0\left(\left(\frac{\lambda_{2}}{r}\right)^{t}\right)\right] .
\end{aligned}
$$

In this case $q(t)$ is asymptotic to a function that changes sign in every time interval of length $\pi / \theta$, and $\pi / \theta \leqslant j+1$.

\section{AN EXAMPLE WITH NO UPPER AGE LIMIT}

We consider the discrete model that most nearly corresponds to the continuous model of Nagylaki (1975). Let

$$
\begin{aligned}
& l(x)=\alpha^{x}, \text { where } 0<\alpha<1 \\
& i=0, \text { and } j=k=\infty .
\end{aligned}
$$

The assumption is that, of the individuals of any sex-genotype category alive in age group $x$ at time $t$, the fraction $\alpha$ of them survive to age group $x+1$ at time $t+1$, that there is no upper bound on the age of an individual, and that matings occur in all age groups. The quantities $A$ and $B$ are calculated as

$$
A=B=\sum_{x=0}^{\infty} \alpha^{x}=1 /(1-\alpha)
$$

and

$$
\begin{aligned}
q(t+1) & =(1-\alpha)\left\{-\frac{1}{2}(1-\alpha) \alpha^{0} \sum_{x=0}^{\infty} \alpha^{x} y(t-x)+\sum_{x=1}^{\infty} \alpha^{x} y(t-x+1)\right\} \\
& =(1-\alpha)\left\{-\frac{1}{2}(1-\alpha) \sum_{x=0}^{\infty} \alpha^{x} y(t-x)+\alpha \sum_{x=0}^{\infty} \alpha^{x} y(t-x)\right\}
\end{aligned}
$$




$$
\begin{aligned}
& =(1-\alpha)\left(\frac{3}{2} \alpha-\frac{1}{2}\right) \sum_{x=0}^{\infty} \alpha^{x} y(t-x) \\
& =\frac{1}{2}(3 \alpha-1) q(t) .
\end{aligned}
$$

Then

$$
q(t)=\left[\frac{1}{2}(3 \alpha-1)\right]^{t} q(0) .
$$

Because $0<\alpha<1,-1<-\frac{1}{2}(3 \alpha-1)<1$ so that $q(t)$ converges to zero, as is expected, and the type of convergence is distinguished by two conditions.

(i) If $0<\alpha<\frac{1}{3}, \frac{1}{2}(3 \alpha-1)$ is negative, and $q(t)$ oscillates between positive and negative in each time interval as it approaches zero.

(ii) If $\frac{1}{3}<\alpha<1, \frac{1}{2}(3 \alpha-1)$ is positive, and $q(t)$ converges monotonically to zero.

A small value of $\alpha$ is associated with little overlapping of generations (the proportion of the total population in age group zero is $1-\alpha$, and the proportion in all other age groups is $\alpha$ ). In the extreme case of $\alpha=0$, there is no overlap of generations, and the standard result $q(t+1)=-\frac{1}{2} q(t)$ of discrete time non-overlapping generations is obtained. If one were to use this model for a natural population with truly overlapping generations and the parameter $\alpha$ were less than $\frac{1}{3}$, a better model might be obtained if the time interval were reduced (halved, for instance) and $\alpha$ were replaced by a larger quantity $(\sqrt{\alpha}$ in this instance). As the time interval approaches zero, $\alpha$ approaches 1 , and the discrete model approaches a continuous model with death rate $d=\lim (1-\alpha) / \Delta t$, and in which $q(t)$ will approach zero monotonically with time.

\section{Discussion}

We believe that the preceding examples illustrate the importance of incorporating age structure in overlapping generation models and provide a way of understanding the distinction between oscillating and monotone convergence of $q(t)$ to equilibrium. In all of the examples with finite upper age $d$ at death, $q(t)$ eventually oscillates between positive and negative values with a period that does not exceed $d$. The models are discrete-time models, but the same result is obtained for the continuous-time analog to (5) and (6) by using theorems of Lotka and Feller on the renewal equation (as contained in Chapter 7 of Bellman and Cooke (1963)). We conjecture that, in natural populations, because there will be finite upper age limits, the quantity $q(t)$ will oscillate with time, although the oscillations may be irregular and difficult to perceive.

Acknowledgment.-The author appreciates the very useful discussions and suggestions of Professors Edward Pollak and Oscar Kempthorne.

\section{ReFERENCES}

Bellman, R., AND COOKE, K. L. 1963. Differential-Difference Equations. Academic Press, New York.

Crow, J. F., AND kimura, M. 1970. An Introduction to Population Genetics Theory. Harper and Row, New York. 
Kempthorne, o. 1969. An Introduction to Genetic Statistics. Iowa State University Press, Ames, Iowa.

LESLIE, P. H. 1945. On the use of matrices in certain population mathematics. Biometrika, $33,183-212$.

NAGYLAKI, T. 1975. A continuous selective model for an X-linked gene. Heredity, 34, 273-278. 